\title{
Preparation of Intracellular Metabolite Extracts from Liquid Schizosaccharomyces pombe Cultures
}

\author{
Tomáš Pluskal,, 1,2,4 Takahiro Nakamura, 1,3 and Mitsuhiro Yanagida1 \\ ${ }^{1}$ G0 Cell Unit, Okinawa Institute of Science and Technology Graduate University (OIST), Onna-son, Kunigami, \\ Okinawa 904-0495, Japan
}

\begin{abstract}
The success of metabolomic analysis relies heavily on the sample preparation protocol. Here we present a protocol for intracellular metabolite extraction from liquid fission yeast cultures based on rapid quenching in pure methanol at $-40^{\circ} \mathrm{C}$, bead-beating in $50 \%$ methanol for cell disruption, and $10 \mathrm{kDa}$ cutoff ultrafiltration for removal of proteins. Samples are concentrated by vacuum evaporation and resuspended in 50\% acetonitrile for mass spectrometric analysis. This protocol is optimal for extraction of polar metabolites such as amino acids, organic acids, nucleotides, sugars, or sugar-phosphates. Its implementation requires $<6 \mathrm{~h}$ and allows preparation of multiple samples in parallel.
\end{abstract}

\section{MATERIALS}

It is essential that you consult the appropriate Material Safety Data Sheets and your institution's Environmental Health and Safety Office for proper handling of equipment and hazardous material used in this protocol.

\section{Reagents}

4-(2-hydroxyethyl)-1-piperazineethanesulfonic acid (HEPES) and piperazine- $N, N^{\prime}$-bis(2-ethanesulfonic acid (PIPES) in $1 \mathrm{~mm}$ stock solutions

These compounds are used as internal standards, not as buffers. Other small molecules can also be applied as internal standards, provided that they are exogenous, stable compounds that can be reliably detected using the selected detection method.

Acetonitrile (HPLC-grade or better) in 50\% solution (v/v)

Methanol (HPLC-grade or better) in $100 \%$ and $50 \%$ solutions (v/v)

$\mathrm{NaOH}(0.1 \mathrm{~N})$

Schizosaccharomyces pombe cell culture (e.g., $2 \times 10^{8}$ cells in EMM2 liquid medium)

$50 \mathrm{~mL}$ of mid-log phase culture is a good starting amount for the first metabolome experiment. In practice, a much smaller amount of cells may suffice, depending on the sensitivity of the LC-MS system and the abundance of target metabolites. For advice on S. pombe culture, see Introduction: Growth and the Environment of Schizosaccharomyces pombe (Petersen and Russell 2016).

${ }^{2}$ Present address: Whitehead Institute for Biomedical Research, Cambridge, Massachusetts 02142.

${ }^{3}$ Present address: Department of Life Sciences, Graduate School of Arts and Sciences, The University of Tokyo, Meguro-ku, Tokyo 1538902, Japan.

${ }^{4}$ Correspondence: pluskal@wi.mit.edu

From the Fission Yeast collection, edited by lain M. Hagan, Antony M. Carr, Agnes Grallert, and Paul Nurse.

(C) 2016 Cold Spring Harbor Laboratory Press

Cite this protocol as Cold Spring Harb Protoc; doi:10.1101/pdb.prot091553 
T. Pluskal et al.

\section{Equipment}

Bead-beating system for cell disruption (e.g., Multi-beads Shocker [Yasui Kikai] or Geno/Grinder [SPEX SamplePrep])

Centrifuge tubes (50-mL, screw-cap)

Centrifugal filters with $10 \mathrm{kDa}$ cutoff, compatible with $50 \%$ methanol solvent (e.g., Amicon Ultra-4

[Millipore UFC801096])

Deep freezer $\left(-40^{\circ} \mathrm{C}\right)$

Forceps

Glass beads (acid-washed [Sigma-Aldrich G8772])

High-speed centrifuge capable of $-20^{\circ} \mathrm{C}$

A centrifuge with a swinging rotor is preferable to a fixed-angle rotor. Both 50- and 15-mL tubes are used.

Microcentrifuge

Microcentrifuge tubes $(1.5-\mathrm{mL})$

Microscope

Needle (26-gauge)

Rotary evaporator

Vacuum filtration equipment consisting of a vacuum pump, collection flask (e.g., Millipore XX1004744), filter holder (e.g., Millipore XX1004730), and methanol-resistant membrane filters of 1- $\mu$ m pore size (e.g., Millipore JAWP04700)

Vortex

This protocol was previously published (Pluskal et al. 2010) and has been widely applied. The workflow is briefly schematized in Figure 1. Samples are prepared from cell cultures in $<6 \mathrm{~h}$; the $10 \mathrm{kDa}$ cutoff filtration and solvent evaporation are the most time-consuming steps. Using this protocol, one individual can prepare 16 samples in one day. When higher samples numbers are required, split sample preparation over multiple days by storing the quenched cells after Step 9.

Sample Preparation

1. For each culture sample, prepare a 50-mL centrifuge tube containing $25 \mathrm{~mL}$ of $100 \%$ methanol. Store the tubes at $-40^{\circ} \mathrm{C}$.

2. Prepare one tube containing $0.5 \mathrm{~mL}$ of $50 \%$ methanol $(\mathrm{v} / \mathrm{v})$ per sample. Store at $-40^{\circ} \mathrm{C}$.

3. For each culture sample, prepare a tube for the bead-beating system (according to the instrument type) and fill with acid-washed glass beads. Store on ice.

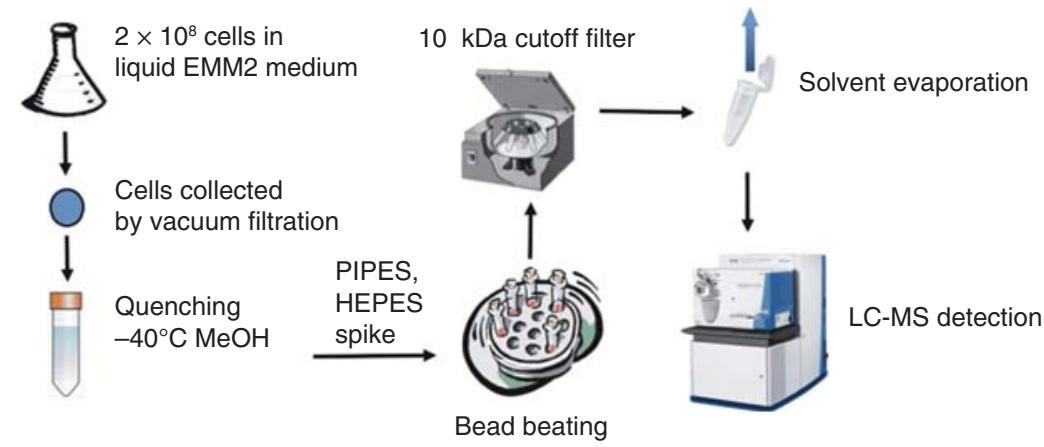

FIGURE 1. Workflow of metabolomic sample preparation. (Reproduced from Pluskal et al. [2010] with permission of The Royal Society of Chemistry.) 
4. Prerinse the $10 \mathrm{kDa}$ cutoff filters according to the manufacturer's instructions.

If the filters are not prerinsed, strong signals of preservatives such as glycerol will be detected in samples. Typically, one round of washing with $0.1 \mathrm{~N} \mathrm{NaOH}$ followed by another round with $\mathrm{H}_{2} \mathrm{O}$ is sufficient. Filters should not be allowed to dry out.

\section{Cell Harvesting and Quenching}

5. Collect all the cells from a culture sample onto a methanol-resistant membrane filter using the vacuum filtration system. Immediately drop the filter (including the cells) into a 50-mL tube containing $25 \mathrm{~mL}$ of methanol $\left(-40^{\circ} \mathrm{C}\right)$ from Step 1 . Vortex the tube briefly to ensure that the filter is completely submerged and place at $-40^{\circ} \mathrm{C}$.

This step should be performed as quickly as possible.

6. After collecting all samples, vortex the sample tubes thoroughly one by one to ensure cells are detached from the filters, and then remove the filters using forceps.

7. Centrifuge the sample tubes at $-20^{\circ} \mathrm{C}(1500 \mathrm{~g}$ for $3 \mathrm{~min}$ should be sufficient), and discard the supernatant.

8. Resuspend each pellet in $0.4 \mathrm{~mL}$ of $50 \%$ methanol $\left(-40^{\circ} \mathrm{C}\right)$ from the tube prepared in Step 2.

9. Add $10 \mu \mathrm{L}$ of internal standard solution (e.g., $1 \mathrm{~mm}$ PIPES and HEPES) to each tube.

Quantities of internal standards can be adjusted according to the measurement method.

After this step, samples can be stored for up to several days at $-40^{\circ} \mathrm{C}$, until the next step is performed.

\section{Cell Disruption and Extraction of Metabolites}

10. Transfer the samples into the appropriate bead-beating tubes prepared in Step 3. Perform bead beating, preferably at low temperature $\left(0^{\circ} \mathrm{C}\right)$.

The efficiency of cell disruption can be verified by microscopy. Alter the beating speed or duration to ensure cell disruption.

11. Collect the fluid from the beads as follows.

i. Puncture the bottom of the bead-beating tube with a 26 -gauge needle and insert the tube into a $1.5-\mathrm{mL}$ microcentrifuge tube.

ii. Centrifuge briefly (e.g,. $700 \mathrm{~g}$ for $1 \mathrm{~min}$ at $4^{\circ} \mathrm{C}$ ) to expel the fluid into the lower microcentrifuge tube, while retaining the beads in the original tube.

Beads can be discarded after use, as consumption per sample is relatively small.

12. Filter the extracted fluid using a prewashed $10 \mathrm{kDa}$ cutoff filter according to the filter manual (e.g., centrifuge at $4000 \mathrm{~g}$ for $60 \mathrm{~min}$ at $4^{\circ} \mathrm{C}$, if using a swing rotor). Use a clean $15 \mathrm{~mL}$ tube to collect the flowthrough. After filtration, discard the filter, including the residual sample (containing proteins and other large molecules).

13. Evaporate the solvent on a rotary evaporator.

Samples can be split into several tubes (e.g., $200 \mu \mathrm{L} /$ tube) to achieve faster evaporation. In order to avoid chemical reactions among the metabolites during evaporation, heat should not be used. Typically the evaporation of $200 \mu \mathrm{L}$ of solvent takes 60-90 min, depending on the instrument.

14. Resuspend the evaporated samples in $40 \mu \mathrm{L}$ of $50 \%$ acetonitrile by gentle pipetting. Store the samples at $-80^{\circ} \mathrm{C}$ until analysis.

The final sample volume and solvent can be adjusted according to the measurement method.

15. Analyze the samples as described in Protocol: Measurement of Metabolome Samples Using Liquid Chromatography-Mass Spectrometry, Data Acquisition, and Processing (Pluskal and Yanagida 2016).

See Troubleshooting. 
T. Pluskal et al.

\section{TROUBLESHOOTING}

Problem (Step 15): During analysis, metabolite signals cannot be detected from samples, or signal intensity is low.

Solution: Check the signals of the internal standards. If they cannot be detected properly, the problem may lie in the detection method. If the internal standards are detected properly, check the efficiency of cell disruption by microscopy. If the signal intensity is low, try increasing culture volume.

Problem (Step 15): Quantitative data are not reproducible.

Solution: Check whether the signal intensity of the internal standards is reproducible. If not, the problem might be with the detection method. If the internal standards are reproducible, make sure the quenching step is performed rapidly, and check the efficiency of cell disruption by bead beating by microscopy.

Problem (Step 15): Quantitative measurements of reduced glutathione (GSH) and oxidized glutathione (GSSG) are not reproducible.

Solution: Glutathione is easily oxidized once exposed to air. Unfortunately, there is no good solution for this problem. For the quantification of glutathione using metabolomics, specialized derivatization methods have been developed (Suh et al. 2009, 2012). However, application of these methods may distort the quantitative measurements of other metabolites.

\section{$\stackrel{\infty}{\circ}$ ACKNOWLEDGMENTS}

We thank Dr. Steven D. Aird for editing the manuscript. We acknowledge the generous funding and support of Okinawa Institute of Science and Technology Promotion Corporation and Okinawa Institute of Science and Technology Graduate University.

\section{REFERENCES}

Petersen J, Russell P. 2016. Growth and the environment of Schizosaccharomyces pombe. Cold Spring Harb Protoc doi: 10.1101/pdb.top079764.

Pluskal T, Yanagida M. 2016. Measurement of metabolome samples using liquid chromatography-mass spectrometry, data acquisition, and processing. Cold Spring Harb Protoc doi: 10.1101/pdb.prot091561.

Pluskal T, Nakamura T, Villar-Briones A, Yanagida M. 2010. Metabolic profiling of the fission yeast $S$. pombe: Quantification of compounds under different temperatures and genetic perturbation. Mol BioSyst 6: 182-198.
Suh JH, Kim R, Yavuz B, Lee D, Lal A, Ames BN, Shigenaga MK. 2009. Clinical assay of four thiol amino acid redox couples by LC-MS/MS: Utility in thalassemia. J Chromatogr B Analyt Technol Biomed Life Sci 877: 3418-3427.

Suh JH, Kim RY, Lee DS. 2012. A new metabolomic assay to examine inflammation and redox pathways following LPS challenge. I Inflamm (Lond) 9: 37. 


\section{Preparation of Intracellular Metabolite Extracts from Liquid Schizosaccharomyces pombe Cultures}

Tomás Pluskal, Takahiro Nakamura and Mitsuhiro Yanagida

Cold Spring Harb Protoc; doi: 10.1101/pdb.prot091553

\begin{tabular}{rc}
$\begin{array}{r}\text { Email Alerting } \\
\text { Service }\end{array}$ & Receive free email alerts when new articles cite this article - click here. \\
\hline $\begin{array}{r}\text { Subject } \\
\text { Categories }\end{array}$ & $\begin{array}{c}\text { Browse articles on similar topics from Cold Spring Harbor Protocols. } \\
\text { Cell Biology, general (1382 articles) } \\
\text { Preparation of Cellular and Subcellular Extracts (104 articles) } \\
\text { Yeast (288 articles) }\end{array}$ \\
\hline
\end{tabular}

